\title{
Choroidal Endothelial Junctions in Primates
}

\author{
A. R. NAGY and T. E. OGDEN \\ Los Angeles, USA
}

\begin{abstract}
Summary
Junctional ultrastructure of endothelial cells of the choroid was studied with freezefracture electron microscopy. A network of multi-stranded linear aggregates was found on arterial endothelial membrane $E$ faces in association with the apposing arterial membrane $P$ face strands. The complexity of the junctional strands decreased as vessel diameter decreased. The choriocapillaris showed staggered junctional strands exclusively on the membrane $P$ face. Junctions of venules and veins were represented by plasmalemmal folds with sparse intramembrane particles on the $P$ face. Freeze-fracture cytochemistry with the membrane probe, filipin, revealed two dissimilar membrane domains: one, an area of membrane fluidity at the junctional strands; and the other, identified by the incorporation of cholesterol into the membrane lipid bilayer, a stable membrane domain. The latter was present throughout endothelial membranes, but was especially prominent on the rims of fenestrations of the choriocapillaris.
\end{abstract}

The structural organisation of the choroid is of great interest because the metabolic needs of the outer retina are supplied by the choroid. The general structural organisation of choroidal blood vessels ${ }^{1}$ and other associated structures within the choroid were described in early electron microscopic studies. ${ }^{2}$ However, the role of the intercellular junctions as barriers to permeability of circulating substances, which enter or exit tissue compartments, was poorly understood at that time, and little insight could be gained regarding the significance of their structural heterogeneity. With the availability of ultrastructural tracers, such as horseradish peroxidase, ${ }^{3}$ which presumably mimic the movement of circulating substances within morphological compartments of microcirculation, functional interpretations of the vas- cular junctions of choroidal vessels became feasible. ${ }^{4,5}$ It became apparent that the structural substrate for the diffusion barrier in endothelial cells of certain capillaries are tight junctions or tight junction-like appositions. ${ }^{6}$ However, due to a large variation in the permeability properties of the numerous vessels studied, it was not clear how endothelial cells permitted passage of molecules of certain sizes and prevented passage of others. If junctional permeability among endothelial cells is dependent on the critical features and extent of inter-endothelial junctions, then methods other than the use of ultrastructural tracers might be more applicable. Since freeze-fracture electron microscopy is uniquely suited to expose broad areas of intramembrane specialisations, we chose this method to examine the organisation of interendothelial junctions in

From: Department of Ophthalmology, University of Southern California School of Medicine, and the Doheny Eye Institute, Los Angeles, California 90033.

Supported by grants EY-03040, EY-02061 and EY-03941 from the National Eye Institute, Bethesda, MD.

Correspondence to: Thomas E. Ogden, MD, PhD, Doheny Eye Institute, 1355 San Pablo Street, Los Angeles, California 90033, USA. 
vessels of the choroid. Prior application of the freeze-fracture technique to the study of endothelial membranes in other tissues suggested the presence of a wide variety and sometimes unique intramembrane specialisation in a variety of vessels. ${ }^{7-10}$ Brief attempts have been made to study the junctional features of some of the endothelial cells in the choroid. ${ }^{11,12}$ However, the information resulting from these studies has been incomplete and sometimes conflicting. Accordingly, we examined the junctional ultrastructure in detail using freeze-fracture electron microscopy correlated with electron microscopy of conventional thin sections of endothelial junctions of vascular segments.

In addition, using the polyene antibiotic, filipin, a marker of membrane sterols, and specifically of cholesterol, ${ }^{13,14}$ we conducted freeze-fracture cytochemistry to identify differences in membrane domains of junctional and non-junctional areas of the endothelial membrane in order to gain further understanding of their structural compartments.

\section{Materials and Methods}

Normal eyes from two cynomolgus monkeys (Macaca fascicularis) and a rhesus monkey (Macaca mulatta) were used. Experimental animals were treated in accordance with the ARVO Resolution on the Use of Animals in Research. Eyes were enucleated under general anaesthesia and prepared according to the protocols described below. There were no differences in the choroidal structure between the two species, so the results will be described together.

\section{Electron Microscopy of Thin Sections}

The enucleated eyes were opened immediately at the equator and fixed by immersion in $2 \%$ paraformaldehyde and $2.5 \%$ glutaraldehyde in $0.1 \mathrm{M}$ phosphate or cacodylate buffer (pH 7.4); the latter contained $5 \mathrm{mM} \mathrm{CaCl}_{2}$. After initial fixation for 10 minutes at $37^{\circ} \mathrm{C}$, the tissue was trimmed and fixed for 2-24 hours at $4^{\circ} \mathrm{C}$. We routinely used 100 micron Vibratome sections, which were postfixed for 1.5 hours in $2 \%$ osmium tetroxide in $0.1 \mathrm{M}$ cacodylate buffer and blockstained overnight with $0.1 \mathrm{M}$ acetate buffered $1 \%$ uranyl ace- tate ( $\mathrm{pH} 5.0)$. We also used $1 \%$ tannic acid in cacodylate buffer ( $\mathrm{pH}$ 7.0) on some tissue. The sections were dehydrated and flat embedded in Epon.

The embedded sections were examined under the dissecting microscope and desired areas were cut out and remounted flat on preformed Epon blocks. Thin sections were cut on an ultramicrotome with diamond knives, were picked up on formvar-coated single-slot grids and stained with uranyl acetate and lead citrate.

\section{Freeze-fracture Electron Microscopy}

Alternate Vibratome sections were fixed for two hours and processed for freeze-fracture after a buffer rinse and cryoprotection in $10 \%$ and $25 \%$ buffered glycerol, one hour each. The sections were mounted between flat gold specimen carriers, frozen in the liquid phase of liquid nitrogen-cooled Freon 22 and stored under liquid nitrogen. Fracturing was done with a hinged double replica device in the Balzers BAF 301 unit at a vacuum of $2-4 \times 10^{-6}$ mbar and at a $153 \mathrm{~K}\left(-120^{\circ} \mathrm{C}\right)$ cold stage temperature. Replicas were produced with about $2 \mathrm{~nm}$ platinum at $45^{\circ}$ and $20 \mathrm{~nm}$ carbon perpendicular to the fractured surface by electron beam evaporation. The replicas were released in $70 \%$ methanol, cleaned with bleach overnight, rinsed several times with distilled water and picked up with formvarcoated single slot grids.

\section{Freeze-fracture Cytochemistry}

After ten minutes of initial fixation, tissues were rinsed in $0.1 \mathrm{M}$ cacodylate buffer and rapidly cut into 50 micron sections with a Vibratome. The strips were placed in $2 \%$ glutaraldehyde in $0.1 \mathrm{M}$ cacodylate buffer containing $0.04 \%$ filipin (a gift from the Upjohn Co., Kalamazoo, MI), which was dissolved first in a few drops of dimethyl sulfoxide (DMSO) to give a final concentration of $1 \%$ DMSO. The sections were incubated in a filipin-containing glutaraldehyde fixative ${ }^{15}$ for 18-24 hours in the dark, with agitation. Following incubation the sections were rinsed in cacodylate buffer and prepared for freezefracture, as described above. Controls omitting filipin were also prepared.

All sections and replicas were examined 
with a Zeiss 10B electron microscope at $80_{\mathrm{KE}} \mathrm{V}$, which was calibrated with a diffraction grating replica and catalase crystals.

\section{Results}

The identity of various segments of the choroidal vascular system was established in most cases by comparing the replicas with thin sections from adjacent Vibratome sections. These could be remounted, sectioned and viewed if the information obtained from the freeze-fracture replicas warranted it. This has been very useful for correlating both sets of data for large arteries and veins. In the case of arterioles and venules, however, we still had to rely on fortuitous fracture faces and the knowledge gained by examining large numbers of thin sections.

\section{Arteries of the Choroid}

Many of the large vessels identified in thin sections were approximately $30 \times 50 \mu \mathrm{m}$ in dimension, had an average of two layers of smooth muscle cells in the tunica media and were enveloped by an elaborate basal lamina that often followed a tortuous course, covering both endothelial cells and smooth muscle cells. Smaller arteries were often enveloped with a single layer of smooth muscle (Fig. 1), although asymmetric distributions of single and multiple smooth muscle cells were also seen. The endothelial cells fre-

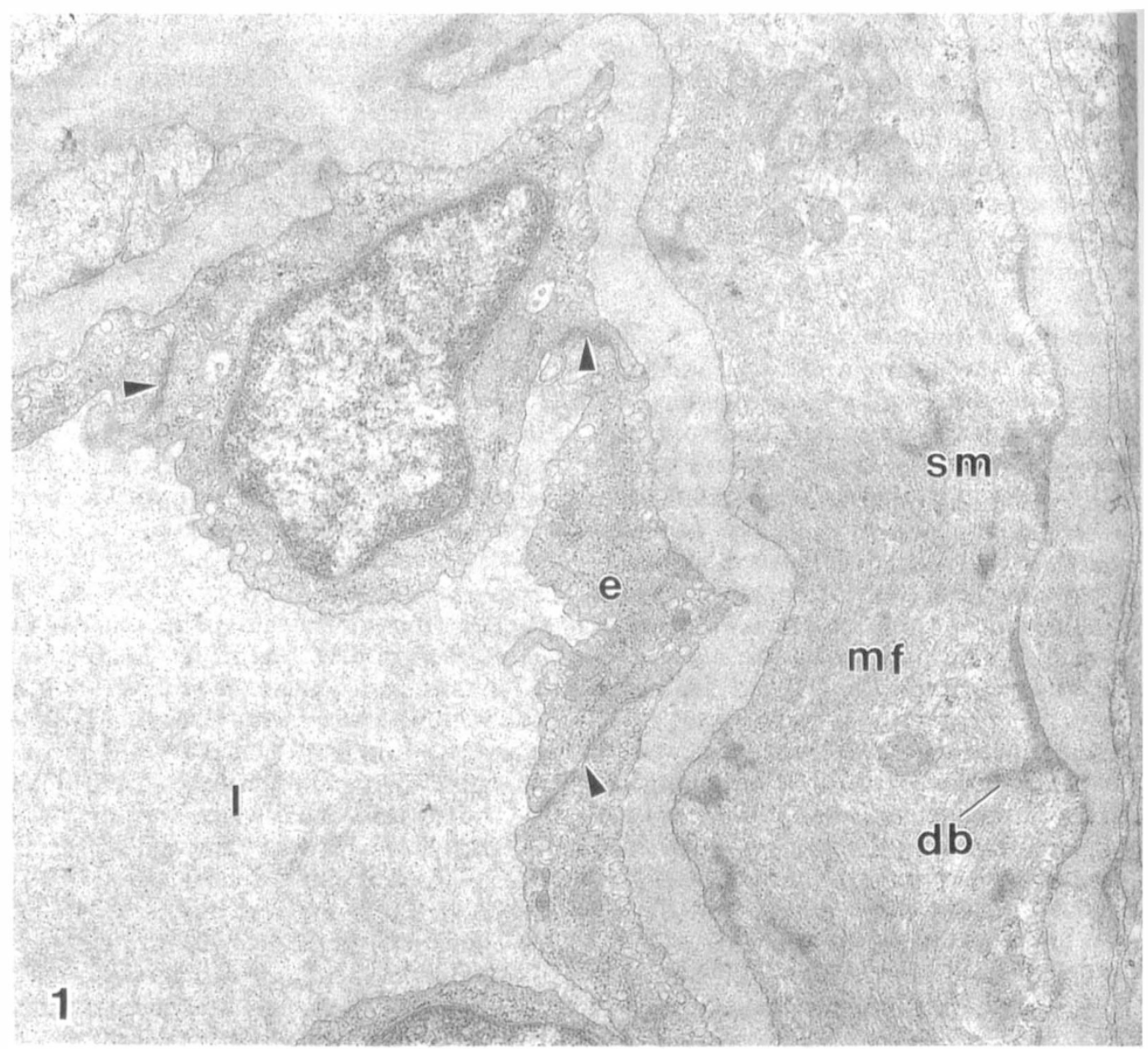

Fig. 1. Electron micrograph of a choroidal artery. Endothelial cells (e) are linked by endothelial junctions (arrowheads). Basal lamina envelops both endothelial and smooth muscle cells (sm). Periodic dense bodies (db) and microfilaments $(\mathrm{mf})$ are notable in the smooth muscle cell cytoplasm. Lumen, $1 .(\times 17900)$. 


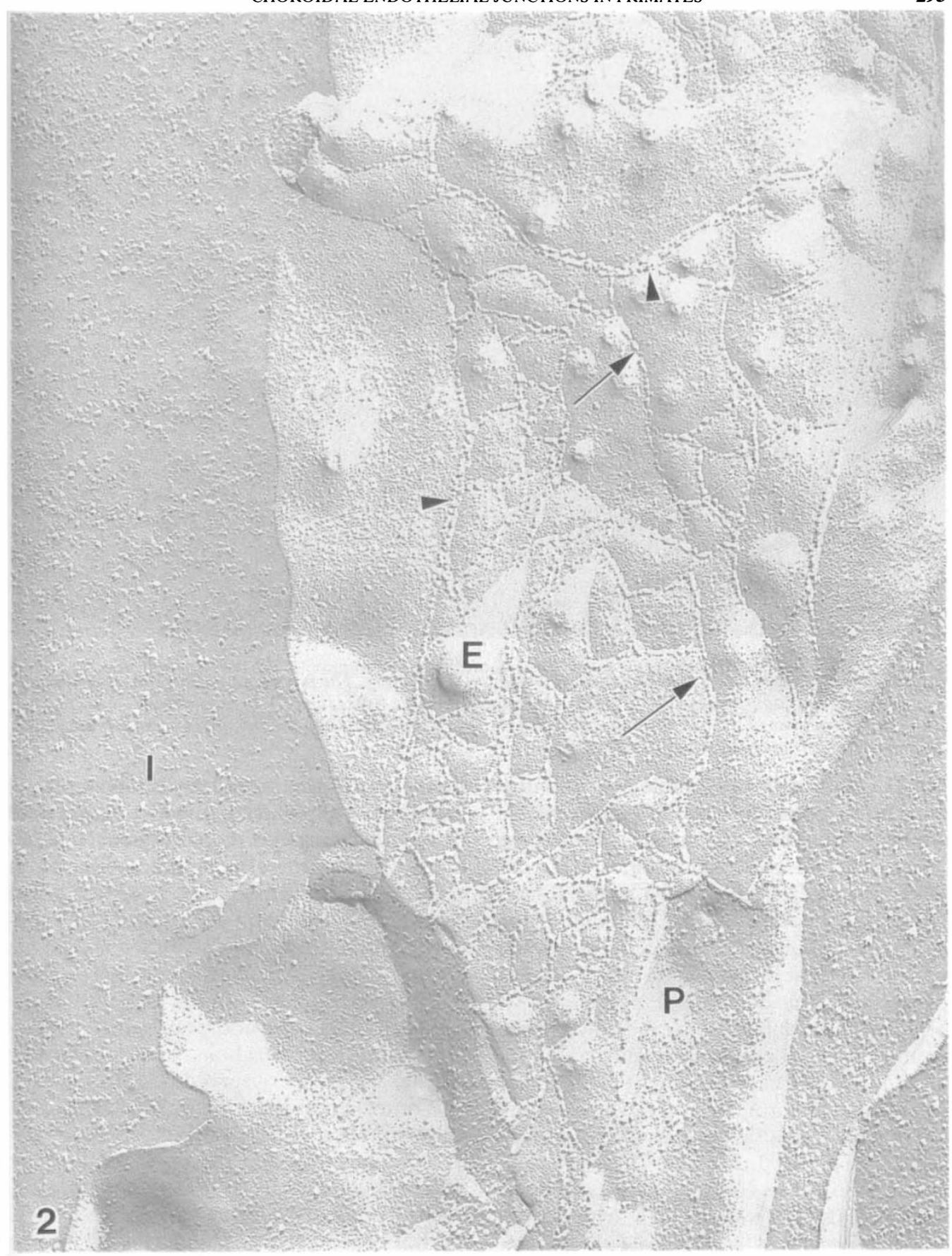

Fig. 2. Freeze-fracture electron micrograph of a junctional zone of a large artery. A complex network compo sed of single (arrows) and double strands (arrowheads) is prominently displayed on the E face (E). P, P face, l, lumen. $(\times 67140)$.

quently showed junctional appositions in thin best observed in freeze-fracture replicas. In sections, but the junctional ultrastructure was large arteries, elaborate junctional strands 


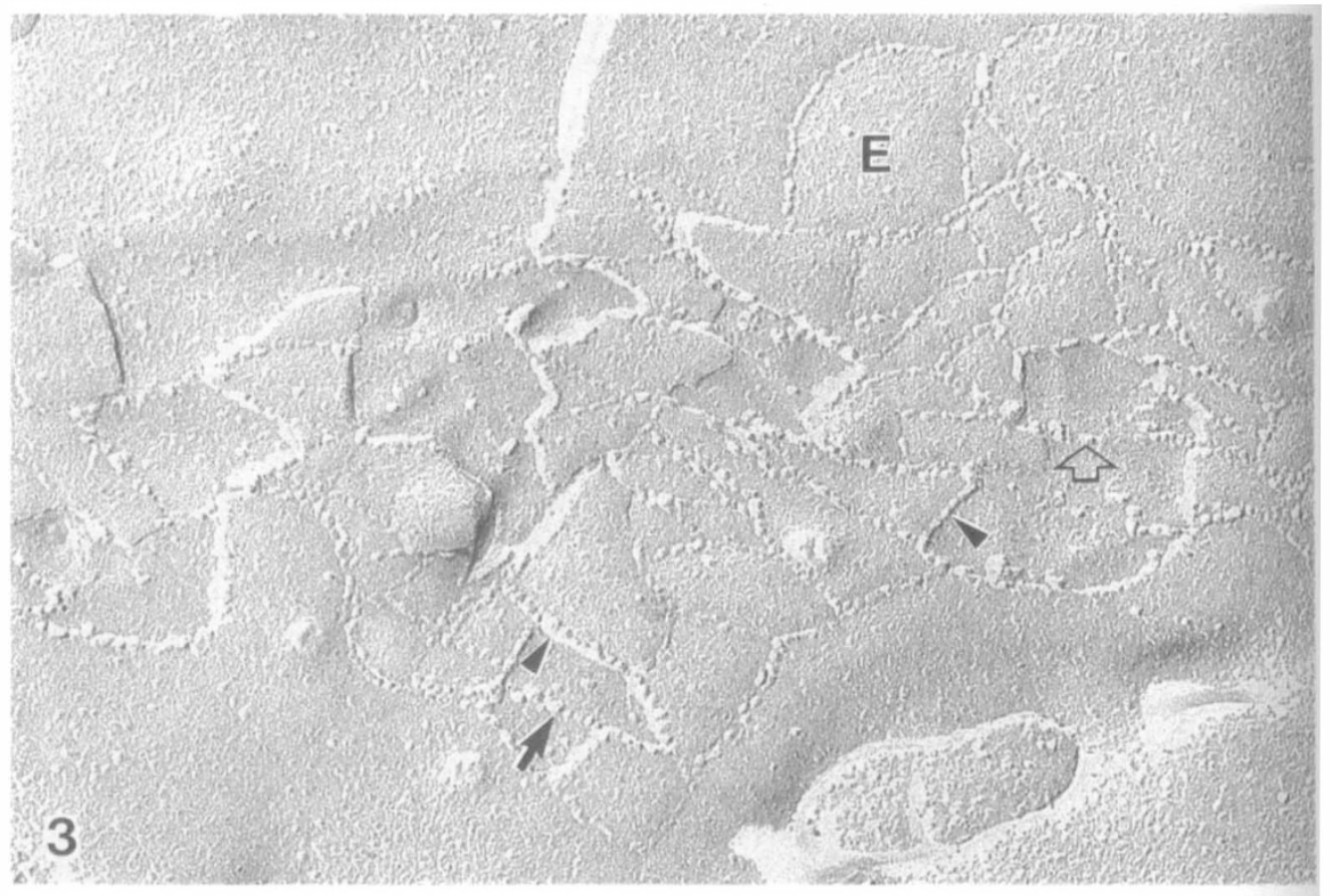

Fig. 3. Freeze-fracture electron micrograph of a medium sized artery. The junctional strands are somewhat less elaborate. A strand composed of closely spaced IMPs appears on the P face (arrow), in register with the overlying $E$ face strand. Remnants of strands are also seen (open arrow). Arrowheads indicate fracturing of membrane halves along junctional strands, E, E face. $(\times 82400)$.

composed of single and double rows of intramembrane particles (IMPs) were visible almost exclusively on the $E$ face; these showed a high degree of anastomosis (Fig. 2). Where the external membrane leaflet is fractured away, it often follows the edge of the junctional strands. Quite often, junctional strands could be observed on the $P$ face in register with the overlying $E$ face (Fig. 3). Remnants of junctional strands that were oriented in a co-linear fashion with strands on the $\mathrm{E}$ face were more frequently observed, suggesting that during the fracture process the IMPs on both membrane faces were removed together (Fig. 3). Interendothelial junctions of arterioles were composed of exclusively single strands, 2-3 strands deep, with a moderate degree of anastomosis (Fig. 4). Occasionally, discontinuous strands and strands with free endings were also noted.

\section{Choriocapillaris}

The 'choriocapillaris, like capillaries in visceral tissues, is highly fenestrated. However, unlike capillaries elsewhere, it possesses a high degree of polarisation with a single layer of vessels facing the retinal pigment epithelium. The lumen, also unlike capillaries in other tissues, is extremely wide $(20-50 \mu \mathrm{m}$ in width). The endothelial cytoplasm of capillaries is rather thin and structural components are most easily observed when the fracture plane is perpendicular to the long axis of the capillary. Due to the abrupt transition of fracture planes, fenestrations and cytoplasmic vesicles are easily observed in both $P$ and $E$ face representations (Fig. 5). Electron microscopy of thin sections of the endothelial junctional zones suggests the presence of tight junctions or zonulae occludentes. However, fusion points of apposing membrane leaflets are not consistently observed (Fig. 6). While in some areas fusion points appear to be relatively frequent, they are lacking in other areas. Goniometric tilt of sections in the electron microscope increased the visibility of membrane fusion points by only $10 \%$; therefore, it appears that capillary endothelial tight 


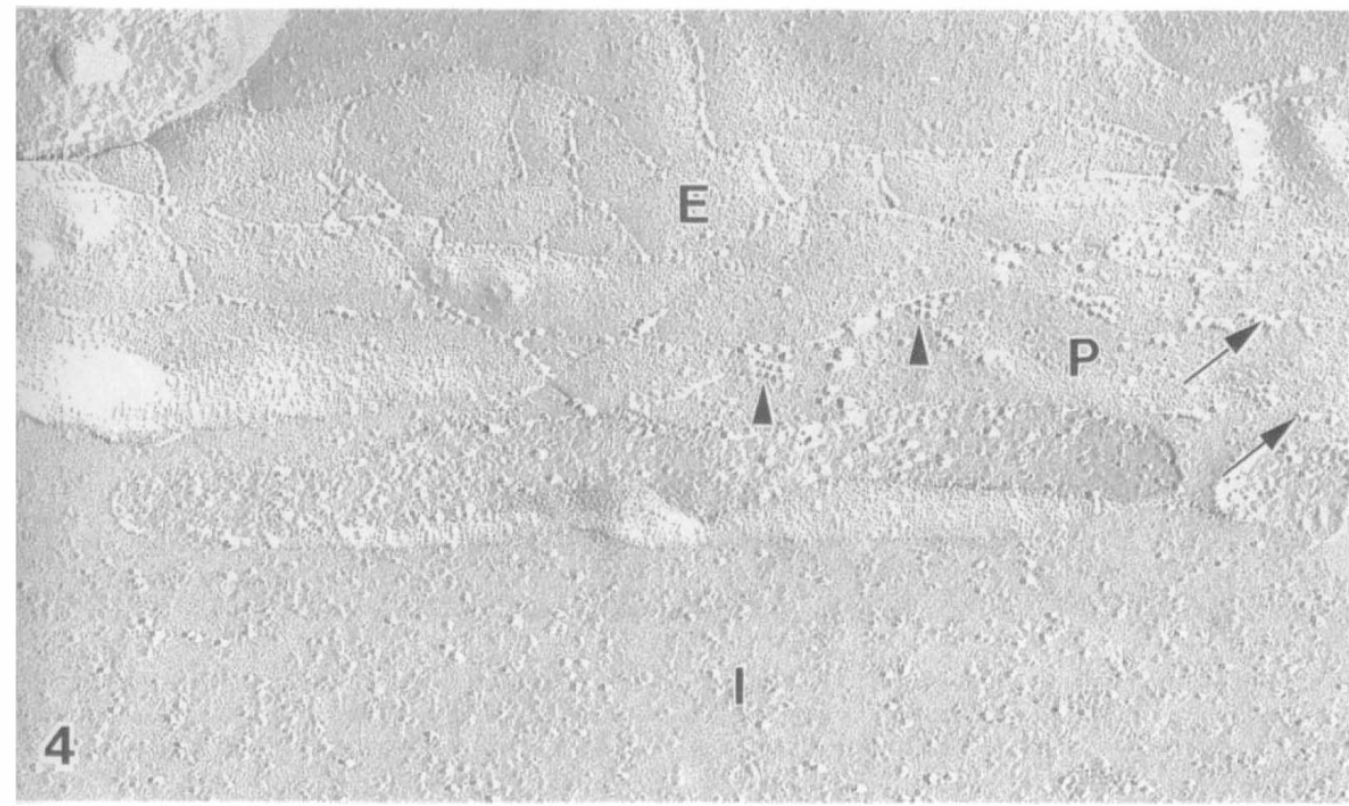

Fig. 4. Freeze-fracture electron micrograph of an arteriolar junction. A single stranded network is seen on the $E$ face (E). Junctional remnants of IMPs (arrows) are evident on the $P$ face $(P)$. Small gap junctions (arrowheads), composed of a few IMPs, are seen at the edges where the fracture plane jumps from the $P$ to $E$ face. l, lumen. ( $\times 82400)$.

junctions frequently have incomplete membrane fusion sites. The interendothelial junctions in freeze-fracture possess junctional strands and discontinuous but closely spaced IMPs on the $P$ face. The rows of junctional strands are staggered, and corresponding

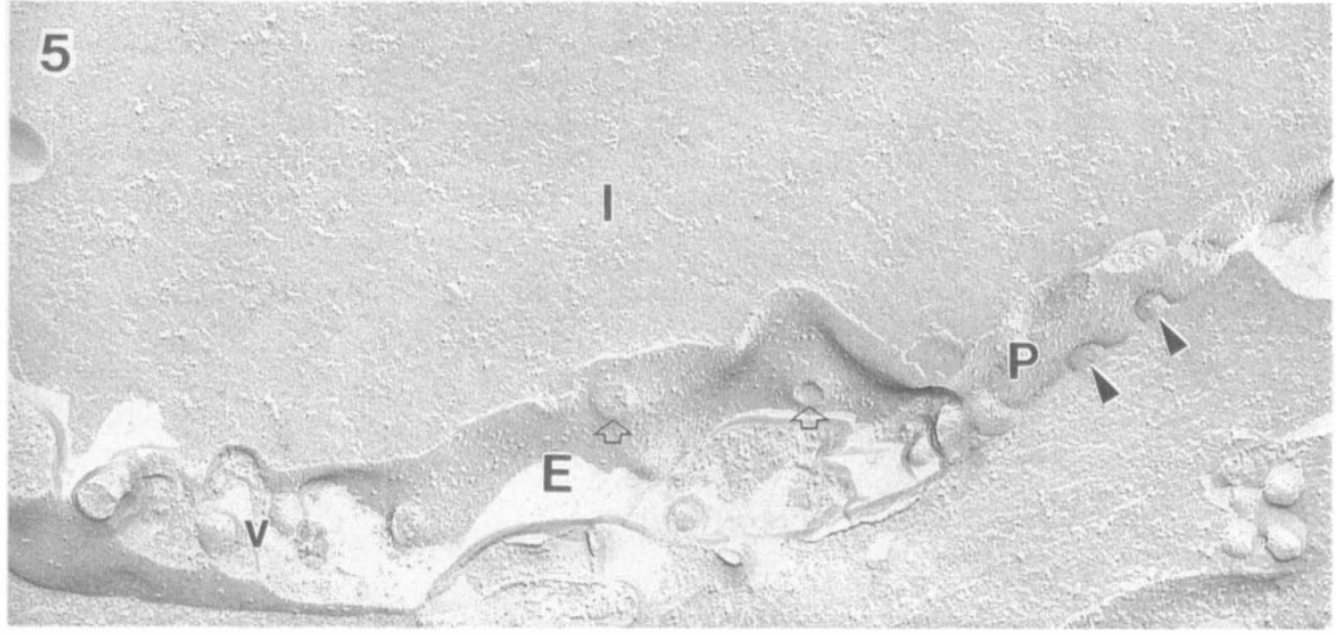

Fig. 5. Freeze-fracture electron micrograph of an endothelial cell of the choriocapillaris. Fenestrations (arrowheads) appear as papillae on the $P$ face $(P)$ or as craters (open arrowheads) on the E face (E). Cytoplasmic vesicles, v. Capillary lumen, $1 .(\times 56280)$. 


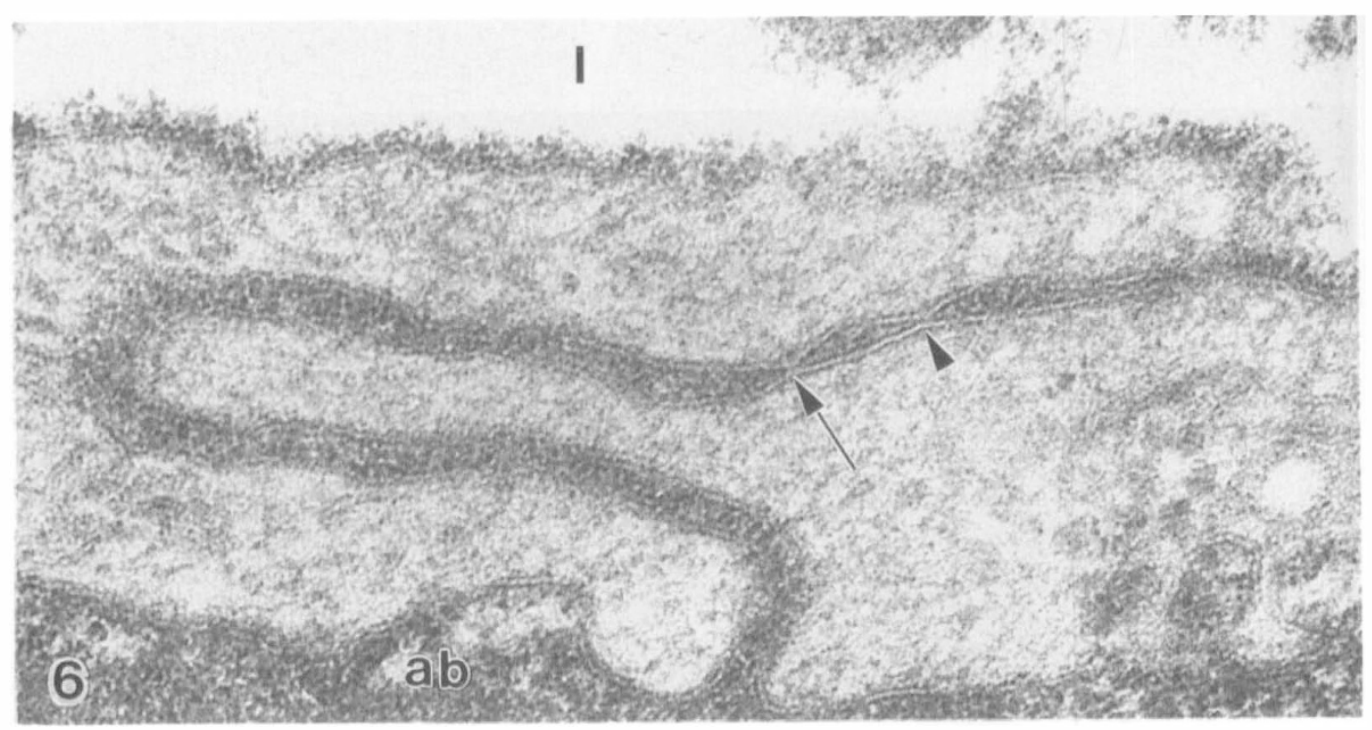

Fig. 6. Electron micrograph of a capillary endothelial junction. A fusion point between apposing membranes is seen (arrow), while the ad jacent membrane apposition (arrowhead) displays an incomplete contact with a narrow gap. The extracellular matrix on the abluminal side $(a b)$ is electron opaque when tannic acid is used. Lumen, 1. $(\times 178200)$.

grooves can be seen on the $E$ face of an adjoining endothelial cell (Fig. 7).

\section{Venules, Veins}

There is a great variation in the cross-sectioned profiles of venules and veins observed in thin sections due to their branching and the tortuous nature of their routes within the choroidal stroma. The endothelium is thin and covered on the abluminal side with basal lamina, which also envelops pericytic processes. The adventitia contains little collagen, but frequently includes thin flat processes of fibroblasts, called veil cells. ${ }^{16}$ An electron micrograph of a small vein is shown in Fig. 8. Freeze-fracture views of junctional areas of veins show a rather simple junctional specialisation; membrane folds that often appear as sharp creases, and which are rarely associated with IMPs at their apex, are seen. The sharp membrane folds seldom anastomose, but have a parallel, staggered appearance (Fig. 9 ). The junctional strands end abruptly, indicating that the junctional specialisation is primarily focal. Venules and small veins show a similar junctional specialisation, although the junctional folds often appear less sharply creased than is the case with the larger veins.

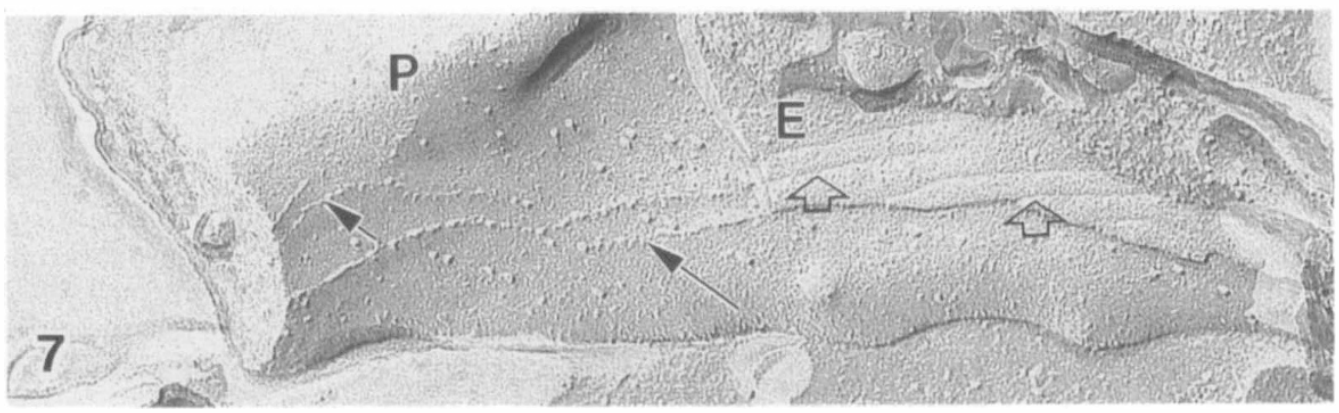

Fig. 7. A capillary junctional zone is seen in a freeze-fracture electron micrograph. Strands and discontinuous rows of IMPs (arrows) are evident on the $P$ face $(P)$ and complementary grooves (open arrows) are located on the $E$ face $(E) .(\times 82400)$. 


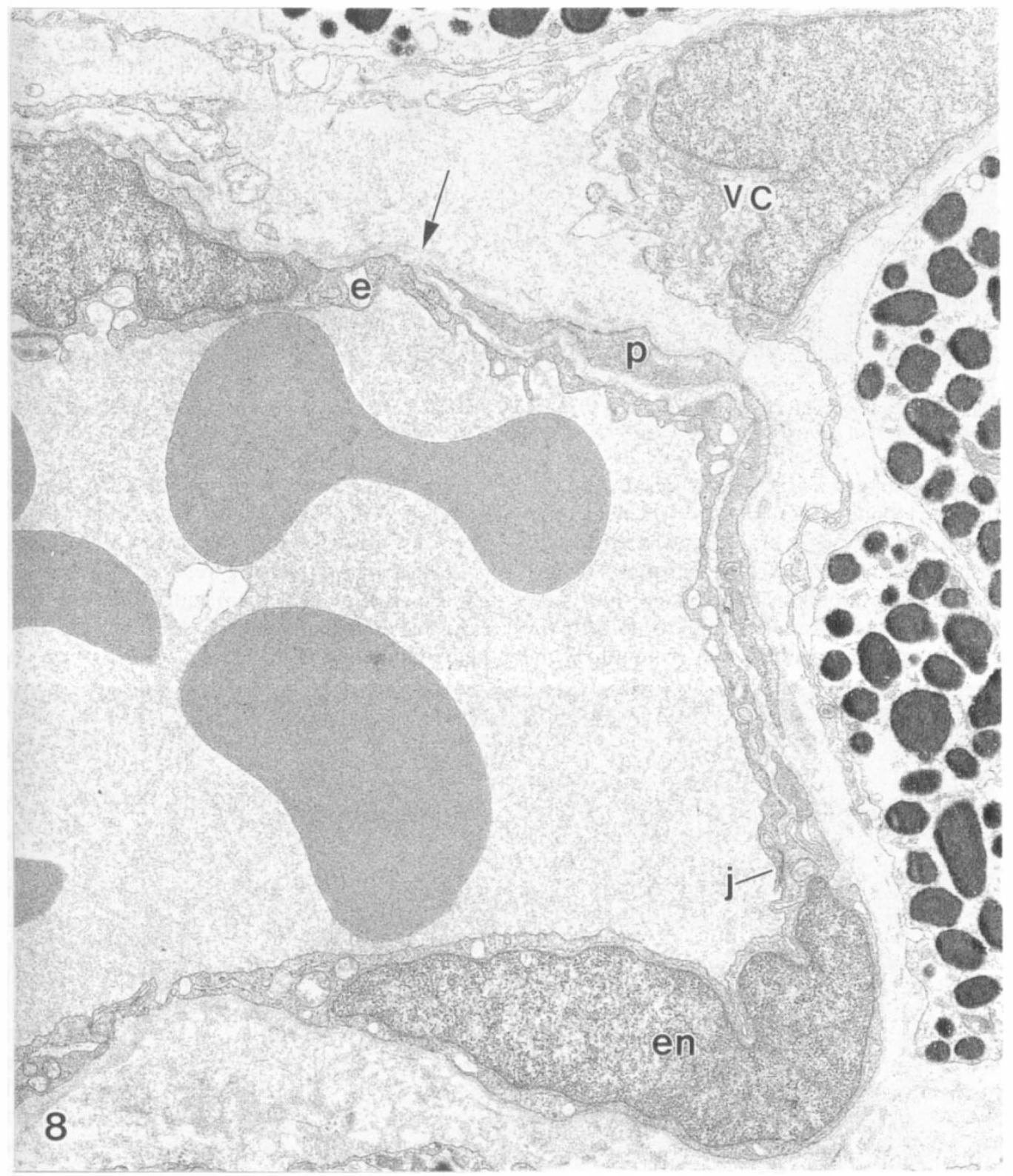

Fig. 8. An electron micrograph depicting the appearance of a small vein. A thin endothelium (e), endothelial nuclei (en) and pericytic processes $(p)$ are seen. Veil cell and its processes (vc). Junctional zone, $j$. Basal lamina, arrow. $(\times 11220)$.

\section{Freeze-fracture Cytochemistry}

The examination of the distribution of filipinsterol complexes on freeze-fracture replicas revealed unequal distribution in labelling. Filipin-sterol complexes are recognised as approximately $25 \mathrm{~nm}$ diameter membrane perturbations that usually appear as protuber- ances on the cytoplasmic membrane-half (P face). Corresponding depressions or invaginations on the $E$ face are also frequently evident. While large areas of the endothelial membranes of arteries, capillaries and veins were labelled, often heavily, with filipin, the labelling was quite limited or absent near the 


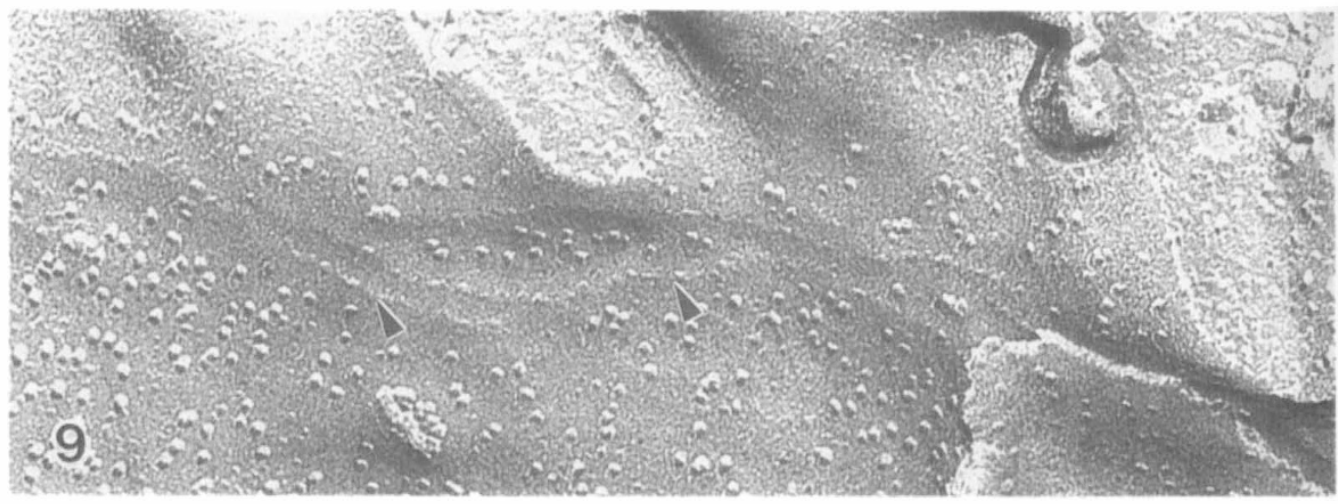

Fig. 9. Freeze-fracture electron micrograph of the junctional area of a vein, composed of staggered membrane creases. Note that IMPs are not associated with the crests of the junctional membrane specialisations. $(\times 106530)$.

parajunctional areas. It was especially notable that no labelling was found on or near junctional strands, and labelling was extremely rare between strands (Fig. 10). Luminal membranes were labelled less densely than were abluminal membranes or membranes of the cytoplasmic protrusions away from junctional folds. Another feature noted with filipinlabelling techniques is the decoration of the rim of fenestrations with the filipin-sterol complexes, occasionally resulting in a uniformly thickened ring around the fenestral rim. The endothelial membrane itself was also heavily labelled (Fig. 11).

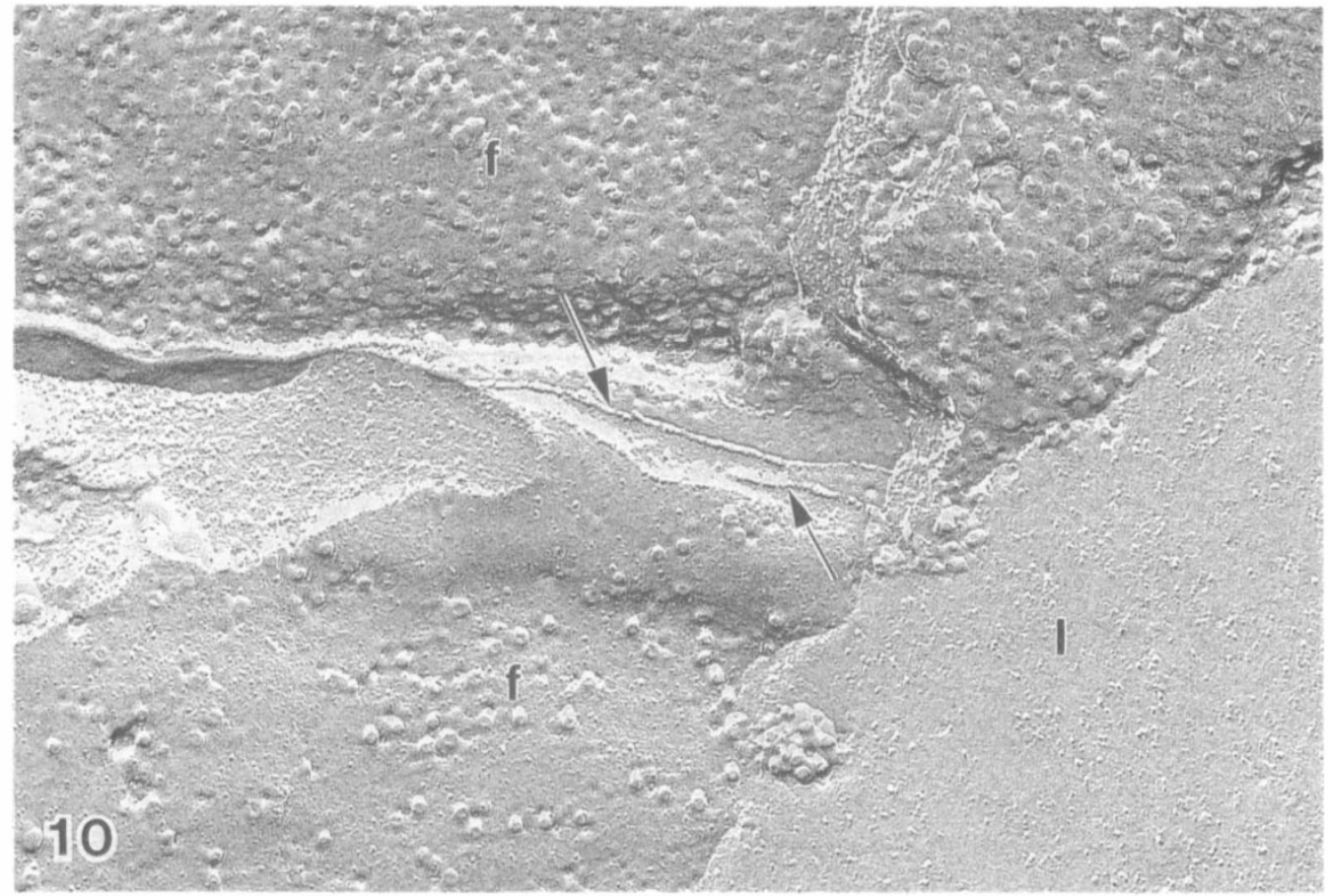

Fig. 10. Freeze-fracture electron micrograph showing the junctional zone and the artery after filipin labelling. Relatively moderate labelling $(f)$ is seen on the luminal endothelial membrane $P$ face, while no labelling is observed on or immediately proximal to junctional strands (arrows). Endothelial membrane in the upper half of the picture is heavily labelled $(f)$ in a uniform fashion, Lumen, 1. (×56 200). 


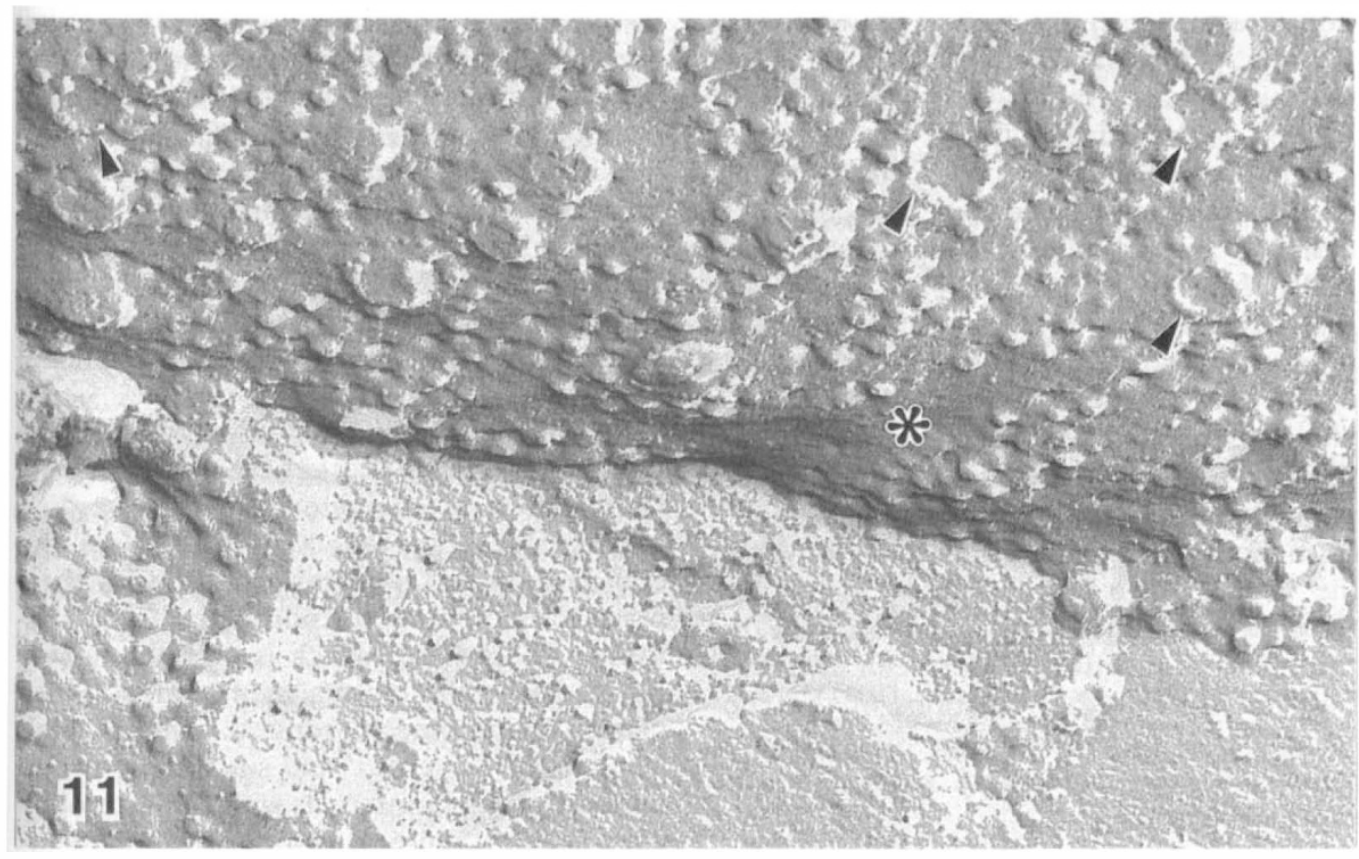

Fig. 11. Filipin-sterol complexes on the endothelial membrane are seen in this freeze-fracture electron micrograph. Heavy labelling with occasional clear patches (asterisk) are seen on the $P$ face. Rings of the filipinsterol complexes are seen around fenestral openings (arrowheads), resulting in a thickened rim. $(\times 81200)$.

\section{Discussion}

Arteries in the choroid exhibit elaborate junctional specialisations, and also segmental variations in the complexity of their junctional ultrastructure. Large arteries possess often multiple junctional strands, a high percentage of which anastomose. While the junctional strands are displayed predominantly on the $\mathrm{E}$ face, fortuitous fracture faces revealed colinear $P$ face strands underlying the $E$ face junctional structures, suggesting that an equally complex network is present on both fracture faces. The fracturing properties of both $\mathrm{E}$ and $\mathrm{P}$ face junctional strands suggest $\mathrm{a}$ very tight coupling of arterial endothelial cells, especially in the case of large arteries. However, the available data regarding permeability properties of arterial junctions is equivocal with respect to their barrier function. This invariability might be related to regional variations in permeability properties in different arteries, ${ }^{17}$ or along different arterial segments. ${ }^{18}$ There also may be limited areas of uniform permeability involving the transcellular route via plasmalemmal vesicles. ${ }^{19}$ Since no careful tracer work has been done to examine in detail the permeability of various types of choroidal vessels, extrapolation of findings from dissimilar tissues would be questionable.

Correlation of tracer data with complexity of junctional strands revealed by freeze-fracture is also difficult, as freeze-fracture reveals the membrane interior, rather than the true external membrane surface that is in contact with solutes. However, the co-linear apposition of both $\mathrm{P}$ and $\mathrm{E}$ face IMPs, and their strong interaction, might prevent patency of these junctions. A similar structural organisation of arterial junctions has been reported elsewhere. ${ }^{10}$ On the basis of the data presented here, it might be reasonable to conclude, on structural grounds above, that at least large arteries are tightly coupled. This may not be the case for smaller arteries and arterioles. The degree of complexity of junctional strands appears to decrease with decreasing vessel diameter, with the smallest vessel displaying single strands, fewer anastomoses, and occasional free endings among strands. The only other description of choroidal arteries closely resembling the descrip- 
tion of arterioles presented here was that of Raviola. ${ }^{12}$ However, our findings differ in that the junctional strands were preferentially located on the $\mathrm{E}$ face.

Endothelial junctions of the choriocapillaris are similar to those described by others, ${ }^{11,12}$ although the prominent folds with rows of IMPs observed on the top of the crests were rarely seen. While the visualisation of membrane folds is dependent on the shadow angle of the platinum with respect to the fracture plane, a large number of replicas studied here showed a more conventional appearance: parallel rows of strands alternating with discontinuous IMPs on the $P$ face and grooves on the $\mathrm{E}$ face. The correlation of incomplete fusion points seen in thin sections with the discontinuity of junctional IMPs seen in freezefracture suggests that junctions of the choriocapillaris are leaky. This suggestion is supported by permeability studies on the choriocapillaris. ${ }^{4}$

The contributions of the capillary fenestrations in the passage of substances, in general, had been obscure until recently. With quick freeze, deep-etch methods, wedge-shaped channels were found between fibrils radiating from the central thickening of the diaphragm to the rim of the fenestrations. ${ }^{20}$ These channels, with an octagonal symmetry and a width of about $5.5-7.5 \mathrm{~nm}$, are permeant to horseradish peroxidase $(4.5 \mathrm{~nm}$ dia) but not ferritin (11 nm dia). Therefore, the diaphragms in fenestrations fulfill the requirements of the small pore system (appr. $6 \mathrm{~nm}$ dia), a physiological concept previously hypothesised..$^{21,22}$

Venules and veins were characterised by the simplicity of the freeze-fracture appearance of their interendothelial junctions in this study. The junctional folds were either moderately sloping structures with few, if any, IMPs associated with the crests of the membrane folds, or sharp creases. Few, if any, differences were noted between the junctional architecture of venules and veins, except with the increase of luminal diameter the junctional folds were somewhat longer and the membrane creases sharper. Venular junctions of other tissues had a similar freeze-fracture appearance. ${ }^{9}$ By contrast, junctional structures ascribed to venules of the human choroid were previously interpreted as being discontinuous zonulae occludentes. ${ }^{11}$ The correct localisation of these junctions on the venular surface was, however, uncertain in that study. The junctional structures described here bear no resemblance to tight junctions, but, rather, are distinct interendothelial junctions specifically associated with venules and veins. The permeability properties of venules to tracers of various sizes would suggest that they function as part of a small pore system. ${ }^{23}$

Finally, junctional and nonjunctional membrane domains of endothelial cells display distinct structural characteristics as described above. Does this reflect differences in the composition and physical properties of their plasma membranes? Endothelial cells synthesise cholesterol, which is probably incorporated into the plasma membrane. ${ }^{24}$ Since plasma membrane in general contains high levels of cholesterol, ${ }^{13}$ and since the polyene antibiotic, filipin, interacts with cholesterol by altering the physical properties of membranes $^{25,26}$ with perturbations detectable by electron microscopy, this agent has been widely used as a membrane probe, including endothelial cell membranes. ${ }^{27,28}$ The application of filipin to choroidal endothelial membranes resulted in the visualisation of two distinct membrane domains: (1) the absence of labelling in the immediate vicinity of the junctional strands of arteries, arterioles, capillaries and venules and (2) the heavy labelling of all other endothelial membranes. The rim of the fenestral openings was also labelled, displaying a thickened ring-like appearance of filipin-sterol protuberances around the perimeter. Similar labelling has also been observed by Simionescu et al. ${ }^{28}$ who also claimed partial labelling of the intrastrand areas of endothelial junctions after relatively short (60 min.) exposure. In our study, the entire parajunctional zone showed a rather homogeneous absence of filipin-sterol complexes, in contrast to uniform heavy labelling of endothelial membranes elsewhere, except around large protrusions or depressions representing coated pits. ${ }^{29}$ The lack of filipinsterol complexes at the junctional zones and their abundance elsewhere, as observed in this study, suggests the division of the endo. thelial membrane into two distinct domains, each with different properties of membrane 
fluidity. ${ }^{13,28}$ The low cholesterol content of junctional zones, as evidenced by the lack of filipin-sterol complexes, would increase membrane fluidity. This, in principle, would permit rearrangement or formation of new junctions. Conversely, the higher phospolipid content at junctional zones would provide material readily for the formation of junctional strands, which are believed by some to be composed of inverted cylindrical lipid micelles. ${ }^{30}$ The high cholesterol content of endothelial membranes away from the junctional zones, on the other hand, could act as a membrane stabiliser. The result of this might be to make the rim of fenestral openings rigid structures, not free to float in the plane of the membrane in Brownian motion.

In summary, we have described a distinct structural organisation of endothelial junctions of arteries, capillaries and veins of the choroid. Arteries are composed of complex junctional networks on both halves of the apposing endothelial membranes, consistent with low levels of permeability. Choroidal capillaries and veins, on the other hand, are composed of staggered intramembrane strands or simple membrane folds with few IMPs, respectively, consistent with great permeability. This study also provides data on the difference in membrane composition of junctional and nonjunctional membranes, as revealed by freeze-fracture cytochemistry.

The authors thank Dr Richard Wood of the Anatomy and Cell Biology Department for the use of his freeze-fracture equipment and for his useful comments during the course of this work. Thanks are due to Kathy Avery and Kate Borkowsky for their help with the primates, Pat Anguiano for typing the manuscript and Ann Dawson for her editing assistance.

\section{References}

${ }^{1}$ Hogan MJ and Feeney L: Electron microscopy of the human choroid. III. The blood vessels. Am J Ophthalmol 1961, 51: 1084-97.

${ }^{2}$ Feeney L and Hogan MJ: Electron microscopy of the human choroid. I. Cells and supporting structures. Am J Ophthalmol 1961, 51: 1057-72.

${ }^{3}$ Karnovsky MJ: The ultrastructural basis of capillary permeability studied with peroxidase as a tracer. $J$ Cell Biol 1967, 35: 213-36.

${ }^{4}$ Shiose Y: Electron-microscopic studies on blood retinal and blood-aqueous barriers. Jpn J Ophthalmol 1970, 14: 73-87.

${ }^{5}$ Peyman GA, Spitznas M, Straatsma BR: Peroxidase diffusion in the normal and photocoagulated retina. Invest Ophthalmol 1971, 10: 181-9.

${ }^{6}$ Bruns RR and Palade GE: Studies on blood capillaries. I. General organization of blood capillaries in muscle. J Cell Biol 1968, 37: 244-76.

${ }^{7}$ Yee AG and Revel JP: Endothelial cell junctions. $J$ Cell Biol 1975, 66: 200-4.

${ }^{8}$ Staehelin LA: A new occludens-like junction linking endothelial cells of small capillaries (probably venules) of rat jejunum. J Cell Sci 1975, 18: 545-51.

${ }^{9}$ Simionescu M, Simionescu N, Palade GE: Segmental differentiations of cell junctions in the vascular endothelium: The microvasculature. J Cell Biol 1975, 67: 863-85.

${ }^{10}$ Simionescu M, Simionescu N, Palade GE: Segmental differentiations of cell junctions in the vascular endothelium: Arteries and veins. J Cell Biol 1976; 68: 705-23.

${ }^{11}$ Spitznas $M$ and Reale E: Fracture faces of fenestrations and junctions of endothelial cells in human choroidal vessels. Invest Ophthalmol 1975, 14: 98-107.

${ }^{12}$ Raviola G: The structural basis of the blood-ocular barriers. Exp Eye Res 1977, 25 (Suppl): 27-63.

${ }^{13}$ Demel RA and de Kruyff: B The function of sterols in membranes. Biochim Biophys Acta 1976, 457: 109-32.

${ }^{14}$ Severs NJ and Robenek H: Detection of microdomains in biomembranes. An appraisal of recent developments in freeze-fracture cytochemistry. Biochim Biophys Acta 1983, 737: 373-408.

${ }^{15}$ Robinson JM and Karnovsky MJ: Evaluation of the polyene antibiotic filipin as a cytochemical probe for membrane cholesterol. J Histochem Cytochem 1980, 28: 161-8.

${ }^{16}$ Rhodin JAG: Ultrastructure of mammalian venous capillaries, venules, and small collecting veins. $J$ Ultrastr Res 1986, 25: 452-500.

${ }^{17}$ Hüttner I, Boutet M, More RH: Studies on protein passage through arterial endothelium. II. Regional differences in permeability to fine structural protein tracers in arterial endothelium of normotensive rat. Lab Invest 1973, 28: 678-85.

${ }^{18}$ Giacomelli F and Wiener J: Regional variation in the permeability of rat thoracic aorta. Am J Pathol 1974, 75: 513-28.

${ }^{19}$ Schwartz SM and Benditt EP: Studies on aortic intima. I. Structure and permeability of rat thoracic aortic intima. Am J Pathol 1972, 66: 241-65.

${ }^{20}$ Bearer EL, Orci L, Sors P: Endothelial fenestral diaphragms: A quick-freeze, deep-etch study. J Cell Biol 1985, 100: 418-28.

${ }^{21}$ Pappenheimer JR: Passage of molecules through capillary walls. Physiol Rev 1953, 33: 387-423.

${ }^{22}$ Bundgaard M: Transport pathways in capillaries-in search of pores. Ann Rev Physiol 1980, 42: 325-36.

${ }^{23}$ Simionescu N, Simionescu M, Palade GE: Open junctions in the endothelium of the postcapillary venules of the diaphragm. J Cell Biol 1978, 79: 27-44.

${ }^{24}$ Fielding PE, Vlodavsky I, Gospodarowicz D, Fielding $\mathrm{CJ}$ : Effect of contact inhibition on the regulation of cholesterol metabolism in cultured vascular endothelial cells. J Biol Chem 1979, 254: 749-55. 
${ }^{25}$ Kinsky SC: Antibiotic interaction with model membranes. Annu Rev Pharm 1970, 10: 119-42.

${ }^{26}$ Norman AW, Demel RA, de Kruyff B, van Deenen LLM: Studies on the biological properties of polyene antibiotics. Evidence for the direct interaction of filipin with cholesterol. J Biol Chem 1972, 247: 1918-29.

${ }^{27}$ Severs NJ: Plasma membrane cholesterol in myocardial muscle and capillary endothelial cells. Distribution of filipin-induced deformations in freeze-fracture. Eur J Cell Biol 1981, 25: 289-99.
${ }^{28}$ Simionescu N, Lupu F, Simionescu M: Rings of membrane sterols surround the openings of vesicles and fenestrae, in capillary endothelium. $J$ Cell Biol 1983, 97: 1592-600.

${ }^{29}$ Montesano R, Perrelet A, Vassalli P, Orci L: Absence of filipin-sterol complexes from large coated pits on the surface of culture cells. Proc Natl Acad Sci USA 1979, 76: 6391-5.

${ }^{30}$ Kachar B and Reese TS: Evidence for the lipidic nature of tight junction strands. Nature 1982, 296: 464-6. 